\title{
A Hybrid Scheme based on Alternative Scalar Leader Election (HS-ASLE) for Redundant Data Minimization in Multi-event Occurrence Scenario for WMSNs
}

\author{
Sushree Bibhuprada B. Priyadarshini ${ }^{\text {a }}$ \\ a Siksha 'O' Anusandhan Deemed to be University, Bhubaneswar, India, bimalabibhuprada@gmail.com
} \begin{abstract}
The current paper reports a hybrid approach namely "Hybrid Scheme based on Alternative Scalar Leader Election (HS-ASLE)" for camera sensor actuation in multi-event occurrence scenario. In the proposed approach, the whole monitored zone gets segregated into multiple virtual sub-compartments and in each of the sub-compartments, one and three scalar leaders are elected alternatively that behave as the representatives of scalars to report event information. During the event occurrence, the event information gets trapped through the scalar leaders in lieu of scalars and the leaders convey the event occurrence information to the respective camera sensors. Pervasive experiment and observation have been ordained to mark the impact of varying the number of deployed scalar sensors and camera sensors individually on various performance parameters in multi-event occurrence ambience. Further, the numerical outcomes attained in terms of number of cameras actuated, coverage ratio, redundance ratio and energy expenditure for camera activation proclaim the effectiveness of our proposed $H S$ $A S L E$ over the other two existing approaches in literature. Moreover, it is marked that our proposed approach attains maximal event region coverage with least camera activation, least redundant data transmission and lowest energy expenditure for camera sensor actuation as compared to two other approaches, which justify the precedence of our proposition over the other existing approaches.
\end{abstract}

\section{Keywords}

Field of View; Depth of Field; coverage ratio; redundancy ratio; WMSNs

\section{Introduction}

Wireless Multimedia Sensor Networks (WMSNs) represent the networks, where two types of sensors are normally employed namely - scalar sensors and video sensors (also called camera sensors). Both the sensors are deployed for ensnaring the environmental condition during the manifestation of any sort of event in the monitored area under consideration. Due to the use of video sensors, WMSNs are employed in many applications viz. habitat tracking, security detection, patient control, chemical monitoring, inventory tracking, etc. The major matter of concern in this field is that the sprinkled sensors cannot be recharged or replaced to extend the lifetime of the network due to their deployment in remote inaccessible zones.

Fig. 1 illustrates a scenario of WMSNs, where multiple sensors (camera sensors and scalar sensors) are sprinkled. The scalars indicated by pink colour represents the scalars residing at the overlapping area. Whenever, event prevails in the region under speculation, initially, it is noticed through all the scalars that inhabit within the event belt. Consequently, these scalars dispatches such information to the corresponding camera sensors within whose purview they reside. 
Being informed by the event ensnaring scalar sensors, the camera sensors collaboratively determine who among them have to be turned on. However, the underlying complication is that during the camera sensor activation, due to the overlapping occurring among the Field of Views (FoVs) of camera sensors, the concerned scalars lying at the superimposed zones inform the similar event data to the corresponding cameras as represented in Fig.1. On behalf of the prevailing information, same data is repeatedly reported by multiple scalars lying at overlapping zone. This gives rise to redundant data transfer owing to reporting of same information multiple times [1,2], which leads to unnecessary energy consumption, unnecessary camera actuation, and bandwidth utilization.

Various research works have been devised time and again for minimizing the redundancy while affording escalated coverage of concerned geographic zone. Distributed collaborative Camera Actuation based on Scalar Count (DCA-SC) [1] is an approach which considers the camera activation based on diminishing order of the respective values of scalar counts. Similarly, Distributed Collaborative Camera Actuation based on Sensing region Management (DCCA-SM) [2] is a scheme that splits the entire geographic area into various sub-compartments. In every sub-compartment, one cluster head gets selected that reports its concerned camera (s) about the prevailing event.

Likewise, a Non-Heuristic scheme is demonstrated [3], which is a distension of [1] and [2], where reduced number of camera actuation occurs for minimizing the data redundancy conjointly with reduction in the amount of energy consumption for camera activation. Moreover, a Centralised cum Sub-Centralised strategy has been depicted in [4], which is an extension of [3] proffered for handling multi-event occurrence. This method involves storage of data in a centralized communication station known as base station that keeps all the information pertaining to the scalars and cameras. Similarly, the notion of cover sets, discussed in [5] helps in tracking all the desired objects. The devised approach dissociates the concerned nodes into cover sets with generation of maximal count of cover sets.

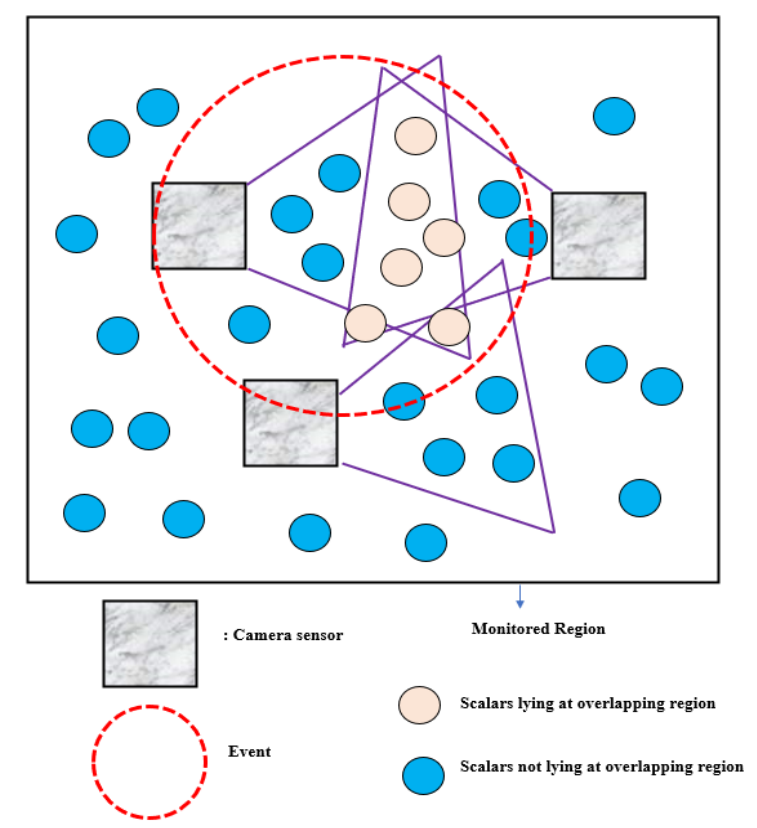

Fig. 1. An Illustration of WMSNs 
The notion of directional region coverage approach is elaborated in [6] that focuses on concerned targets having demarcated priorities. Further, the approach elaborates the priority-based coverage issue that aims to prefer a minimal subset of sensors; those are capable to track concerned destined events to be ensnared while gratifying their corresponding priorities. Similarly, a path-coverage scheme where the details of the coverage phenomenon corresponding to 1-D path is accomplished in [7] through the sensor network which gets modelled as a 2-D Boolean representation. An approach advocated in [8] uses two passes for eliminating the repeated data. In addition, a redundant data elimination technique based on data similarity is presented in [9] that calculates the similarity among the data gathered towards the concerned main station.

Similarly, an optimal approach as discussed in [10] for the deployment of sensor nodes helps in the construction of large networks that get deployed so that this impels the optimal balance between diminishing congestion and routing data packets over shortcut routes. Similarly, the framework depicted in [11] proposes a novel approach for handling huge volume of data from effective devices. A method described in [12] achieves required $k$ coverage and each concerned point is camouflaged by minimum $k$ number of sensors in concerned deployment field.

Moreover, the proposed method frames a maximal count of layers and every layer is 1-covered and 1-connected. Similarly, in [13] an in-depth study got carried out from various perspective deformation states, in which the perspective deformation concerned to a point is segregated into three distinct components. Subsequently, the noise immunity of every part under several FOVs is collaborated to assure the prime element of the hindrance in connection to concerned cameras. Moreover, the method proffered in [14] fetches the Connected Target Coverage predicament having the main aim concentrated on prolonging the network's longevity through ordering the sensors into various sets.

Although several approaches have been manifested as elaborated earlier, still none of those cover the event region properly since while turning on the cameras; there prevails a compromise between the event region covered and number of cameras actuated. This thing occurs because for covering more portions of event area, greater count of cameras has to be activated, that leads to more superimposed area among the FoVs of cameras. Thus, the main objective in such research is to devise a novel algorithm that minimizes such redundant data transmission by activating merely the desired count of cameras while tracking the event zone effectively in a multi-event occurrence scenario.

Our proposed approach elects scalar leaders intellectually and uniformly in alternate virtual sub-compartments such that the cameras that would be activated owing to them will be covering more amount of distinct geographic area of monitored area and side by side diminishing the amount of duplicate data transmittal occurring in WMSNs.

The remaining part of the paper is arranged as follows: Section 2 discusses the proffered approach conjointly with the whole methodology concerned in the proposition. Section 3 depicts the research outcomes attained from the experimental evaluation. In toto, Section 4 concludes the paper. 


\section{Proposed HS-ASLE Hypothesis}

In this research, a novel distributed method namely, Hybrid Scheme based on Alternative Scalar Leader Election (HS-ASLE) for redundant data minimization has been proposed. This algorithm is a hybrid strategy that elects scalar leaders in two distinct manners in alternate subcompartments of the monitored zone, which can effectively handle the multiple event occurrence while actuating least count of cameras for conjointly providing enriched coverage of the ongoing concerned event occurring field.

The steps of our proffered algorithm have been discussed as follows:

\subsection{Step. 1. Initial Deployment of sensors and Virtual Segregation of Monitored Region}

At the outset, all the scalars and camera sensors are sprinkled arbitrarily in concerned tracked zone. Scalars and cameras exchange Scalar Note $(S N)$ and Camera Note $(C N)$ successively. $S N$ and $C N$ represent the messages exchanged by scalar and camera sensors that retain their respective $i d$ and position data. Moreover, the ids associated with all the cameras get reserved in a list namely, My Waiting Catalogue (MWC). Another data structure called as, Actuation list $(A L)$ is there which contains the ids of turned on cameras and this list is initially void.

Subsequently, the sensors estimate the Euclidian distance in between them. The whole monitored zone gets logically splatted into equal-size squared regions so that the length of squared zone is equal to the twice the Depth of Field $(D o F)$ [1] of the concerned camera and each of the smaller regions are known as virtual sub-compartments. Such measure for length selection is chosen for sub-compartment determination to reduce the amount of superimposition among FoVs of the concerned cameras.

\subsection{Step. 2. Scalar Leader (SL) Election in Virtual Sub-compartments}

In every sub-compartment, SL election gets done so that each of the sub-compartment contains at least one Primitive Scalar Leader (PSL) so that it is the least distant scalar present from the centre of the concerned sub-compartment. Likewise, another scalar leader known as Secondary Scalar Leader (SSL) is determined in each of the alternate sub-compartments such that they are the maximum distant scalar present in the concerned sub-compartment from the PSL.

After election of both PSL and SSL, a tertiary Scalar Leader (TSL) gets elected, that indicates the scalar having the minimal mean distance from PSL and SSL. The TSL selection some times becomes impossible for some virtual sub-compartments since there may not be adequate number of scalars for certain sub-compartments since all the sensors get randomly deployed. The selection of three leaders is done so as to cover the event region uniformly along various directions.

In this context, our attempt is to choose three scalar leaders uniformly in alternate compartments so that event region can be uniformly covered by the elected scalar leaders. The approach is a hybrid scheme since in alternative consecutive sub-compartments one and three scalar leaders are elected successively. 


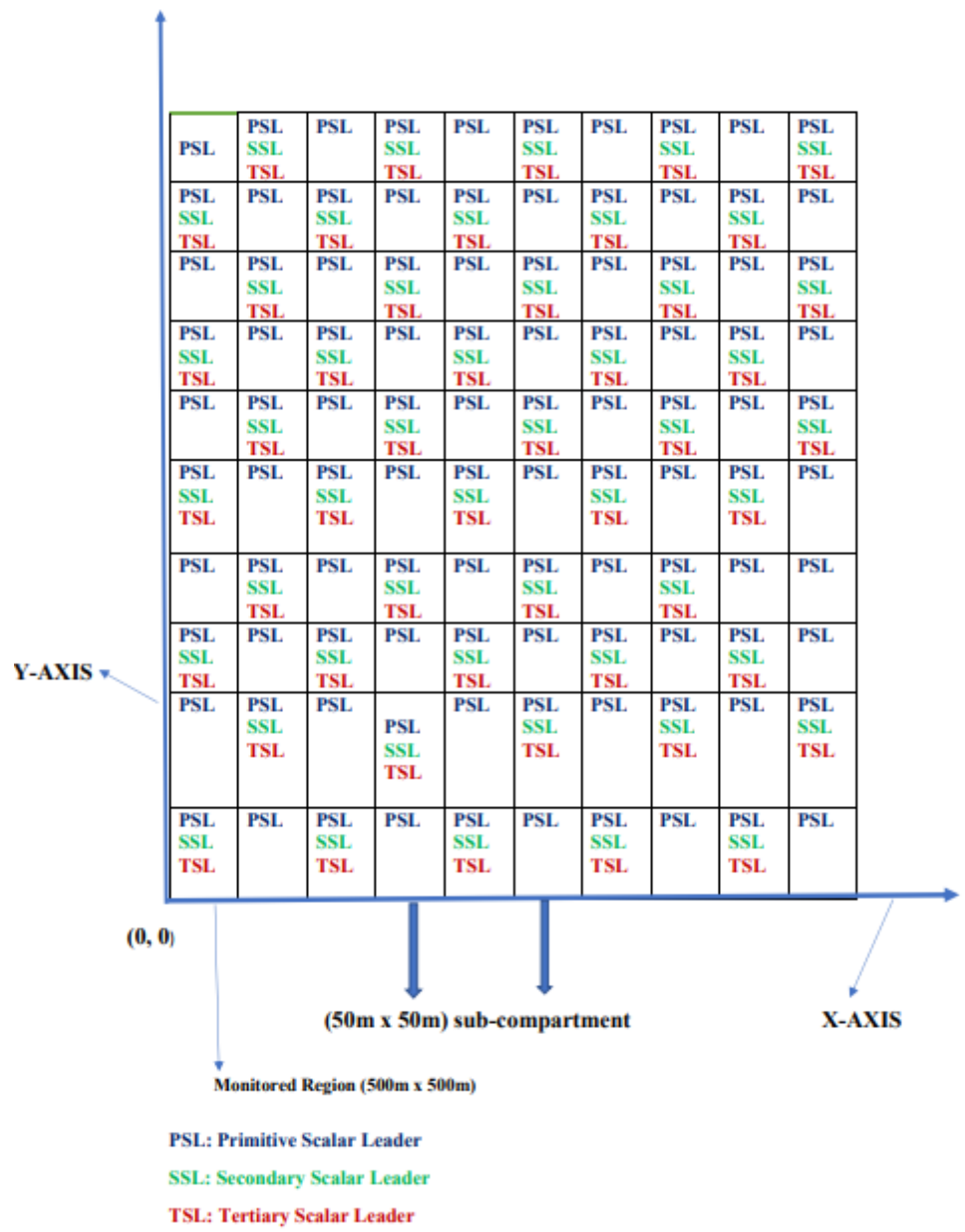

Fig. 2. Splitting of monitored area into logical sub-compartments

Fig. 2 displays scenario of segregation of monitored zone into logical sub-compartments and election of scalar leaders in consecutive sub-compartments. Likewise, Fig. 3 portrays the two detailed scenario of SL selection in consecutive sub-compartments. Since two different strategies are employed in our proffered approach, hence it's a hybrid strategy.

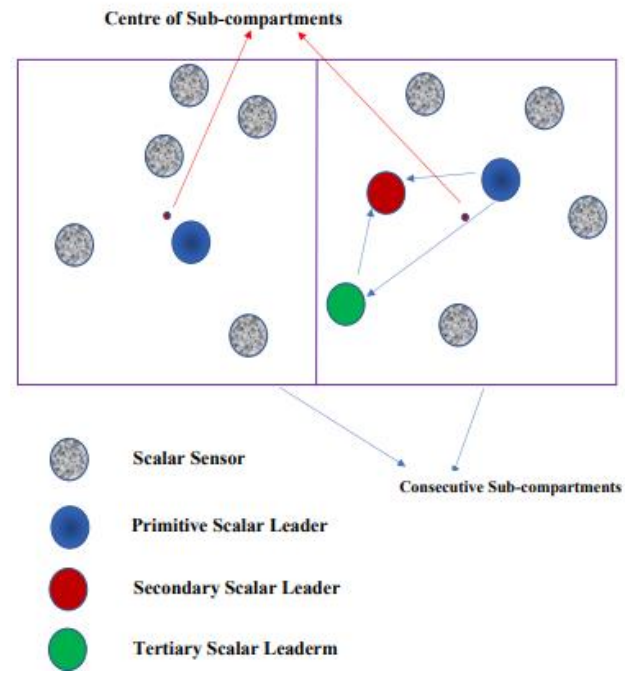

Fig. 3. Detailed Election of SLs in Logical Sub-compartments 


\subsection{Step. 3. Multievent Occurrence, Sensor collaboration and Activation}

Whenever, several events occur simultaneously in a monitored region, they are detected only by the Scalar Leaders (SLs) in each of the squared sub-compartments. A scenario of multi-event occurrence and its detection by multiple SLs has been portrayed in Fig. 4, where we have displayed only four consecutive sub-compartments of the monitored region. Consequently, the premiers communicate such information to the corresponding cameras by sending $M y$ Detect message (MDM). MDM represents the message that retains the ids conjointly with the positional data of the corresponding $S L s$. In case of failure of any $S L$, the nearest $S L$ which is a sensing neighbor of concerned failed node takes part in event information reporting.

Afterwards, the video sensors update the Event Dispatching Scalar Leader List (EDSLL). This list gets retained at every camera containing the event ensnaring scalar leader ids. Thereafter, all the cameras calculate the total count of event ensnaring SLs dwelling within respective DoFs called as Event Dispatching Scalar Leader Sum (ED-SLS).

Three other lists called Multi Leader Camera Catalog (MLCC), Single Leader Camera Catalog (SLCC) and Update Message ID List (UM-IDL) are maintained. MLCC and SLCC are the lists that are preserved by the cameras for keeping the ids of those cameras possessing $E D-S L S \geq 2$ and ER-SPS $\geq 1$ successively in ascending order. Similarly, UM-IDL is a list which holds ids of SLs residing in the Refurbish Scalar Leader (RSL) message of the activated cameras that reserves the ids of SPS dwelling within the DoF of actuated cameras, that are chosen depending upon the estimated Euclidian distance between the leaders as well as the camera.

The camera whose id prevails first in $M L C C$ list is firstly turned on and the concerned id gets included in $A L$ and get removed from $M W C$. During the time when the camera undergoes activation, $U M-I D L$ list gets updated. Subsequently, the activated camera broadcasts ids of scalars present in its FOV by broadcasting RSL message. Afterwards, the subsequent camera dwelling in the $M L C C$ (say, p) compares the ids of $S L s$ held in corresponding ERSLC with the ids of SLs in contained RSL message transmitted via the turned on video sensor. Then the following scenario will get manifested:

\section{If (leader ids contained by USP and in ERSPL match completely)}

Then do not turn on camera $p$

\section{Else}

\section{Camera p undergoes activation}

Likewise, the remaining cameras retained orderly in $M L C C$ list make decision regarding actuation and at the time when, a camera is tuned on, the concerned id gets included in $A L$ and is removed from $M W C$. Once a particular camera is activated, the ids of scalars residing in $R S L$ messages of all the actuated cameras in $U M-I D L$ for $M L C C$ list are compared with the $S L$ ids found in EDSLL of the first camera residing in $S L C C$.

If the $S L$ ids retained in EDSLL of the first camera dwelling in SLCC matches fully with the $S L$ ids retained in updated $U M-I D L$, it is not desired to turn on the corresponding camera. In case, a match is not marked, then the corresponding camera of SLCC is turned on. The turned on camera now broadcasts Refurbish Scalar Leader (RSL) message. 
Subsequently, the remaining cameras present get turned on based on matching their event ensnaring $S L$ ids present in $E D S L L$ with the ids contained in all the $R S L$ messages received from the activated cameras in $U M-I D L$. Finally, the $A L$ is updated which retains the ids of all the activated camera sensors. The diagrammatic representation of the entire working of the proposed approach has been shown in the flow chart portrayed in Fig. 5.

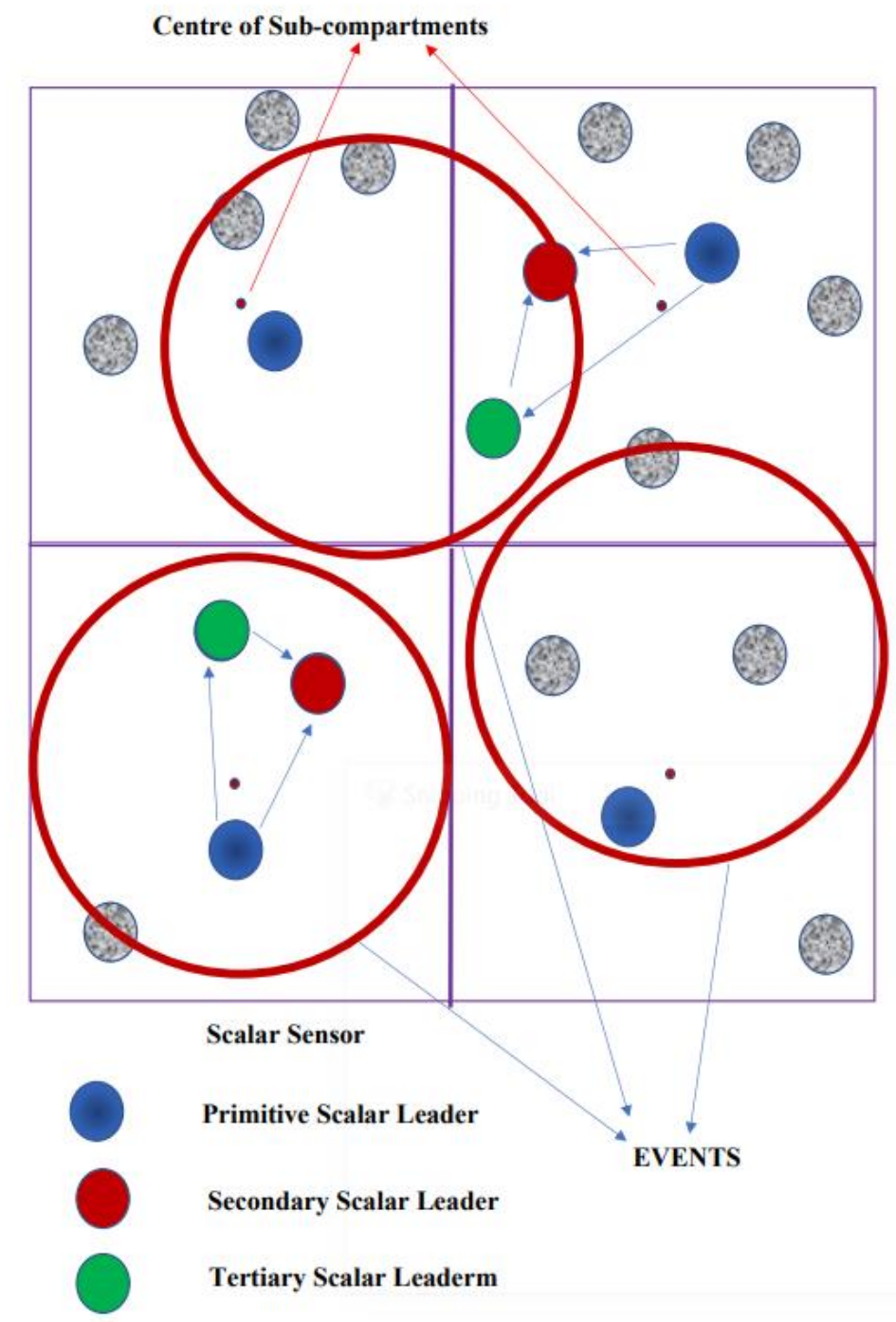

Fig. 4. Multievent Occurrence and Event ensnaring by the Scalar Leaders (SLs) 


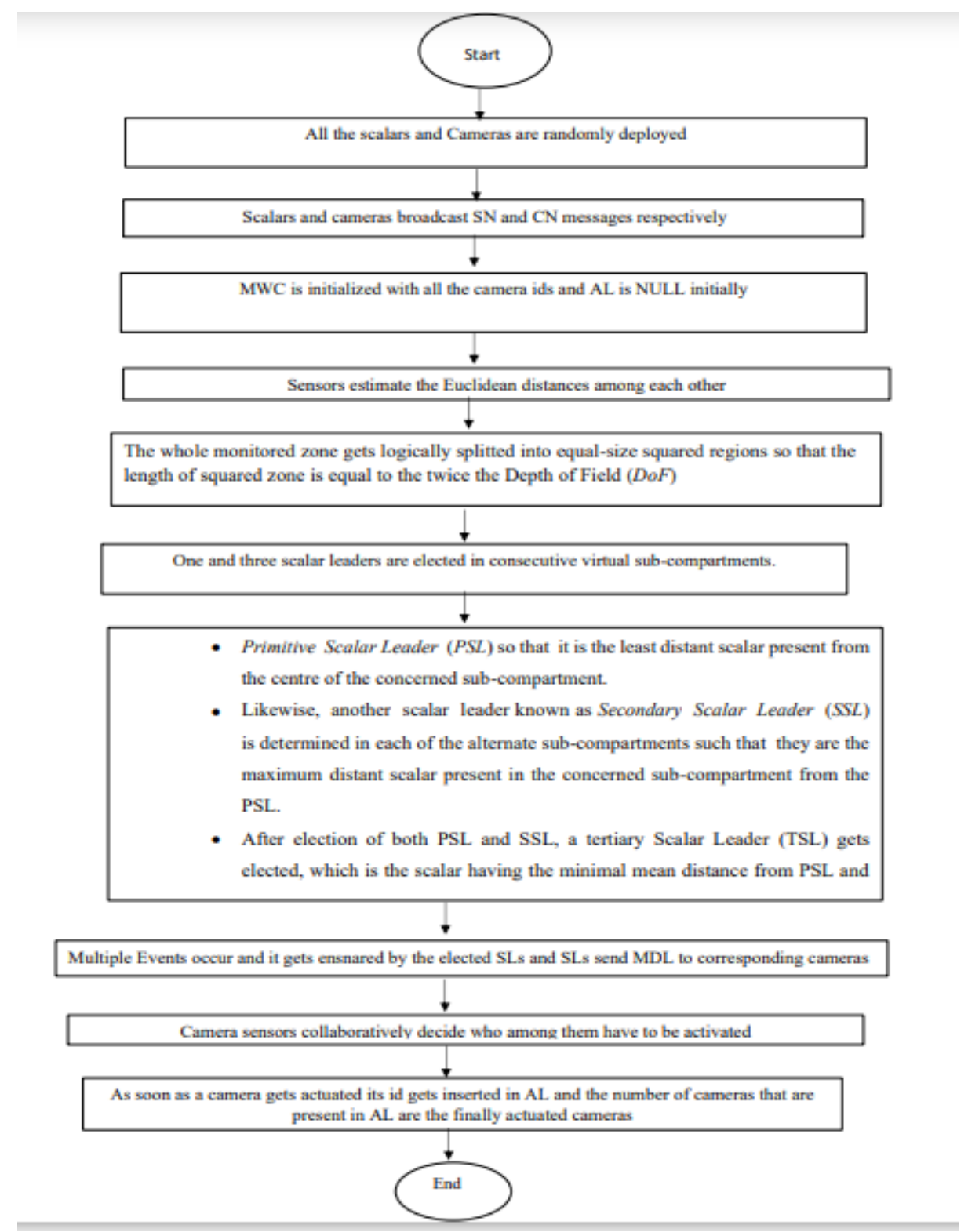

Fig. 5 Entire process of proposed HS-ASLE Approach

\section{Performance Evaluation}

The performance of the proffered algorithm HS-ASLE has been assessed by using $C++$ by conducting comparative assessment with two different schemes - DCA-SC [1], DCCA$S M[2]$.

\subsection{Assumptions}

All the sensors are hypothesized to be sprinkled arbitrarily in a $(500 \mathrm{~m} * 500 \mathrm{~m})$ area . In this research, the same $D O F$ measurement is employed for all the cameras for mollifying the implementation. The number of scalars $(n s)$ and number of cameras $(n c)$ deployed have been varied independently and their repercussion on various performance metrics has been studied as follows:

(i) Number of cameras activated (nca): nca define the total count of cameras which atlength get actuated. Lower is the count of cameras turned on; diminished will be the amount of repeated data transfer. 
(ii) Coverage Ratio (cr): It is "the proportion of area of events covered by actuated cameras to the total area of the prevailing events" [1]. Higher value $\mathrm{cr}$ assures enriched coverage of the ongoing event area.

(iii) Redundancy Ratio (rr): It represents the ratio of whole portions of superimposed zones of FOVs of turned on cameras camouflaging the prevailing event zone to the whole unique parts pertaining to the event zone which get camouflaged by the turned on camera sensors. Lowered value of $r r$ ensures lesser amount of redundant data transmittal.

(iv) Energy Expenditure for Camera Activation (eeca): eeca signifies the amount of energy consumption in the interim of the actuation of cameras. Diminished count of activated camera gives rise to reduced energy expenditure.

The data generation procedure in our proposed approach uses the notations as portrayed in Table 1. Similarly, the data generation procedure is portrayed in Fig. 6.

Table 1. Notations used

\begin{tabular}{|l|l|}
\hline Notations used & \multicolumn{1}{c|}{ Meaning } \\
\hline$n s$ & number of scalar sensors \\
\hline$n c$ & number of camera sensors \\
\hline$n$ and $m$ & number of scalar and camera sensors to be deployed \\
\hline sxc $[\mathrm{i}]$ & array holding $x$ coordinates of scalars \\
\hline syc $[\mathrm{i}]$ & array holding y coordinates of scalars \\
\hline cxc $[\mathrm{j}]$ & array holding x coordinates of cameras \\
\hline cyc $[\mathrm{j}]$ & array holding y coordinate of cameras \\
\hline
\end{tabular}

$/ / \mathbf{x}$ and $\mathbf{y}$ coordinate location generation for sealars

for $(\mathrm{i}=0 ; \mathrm{i}<\mathrm{ns} ; \mathrm{i}++)$

t

sxe[i] $=$ rand $0 \%(\mathrm{n}+1)$;

//sxe[i] is in the range of $\theta$ to $\mathrm{n}$

sycii] $=$ rand $0 \%(n+1)$;

//syc[i] is in the range of $\theta$ to $\mathbf{n}$

cout $<<$ sxe $[i]<* \cdots<<$ sye[i $\mid<<$ endl;

$/ / \mathbf{x}$ and $\mathbf{y}$ coordinate position generation for cameras

forg $0=0 ; \mathbf{j}<$ ne; $\mathbf{j}++)$

exe[j] $=\operatorname{rand} O \%(m+1) ;$

//exelji is in the range of $\mathrm{\theta}$ to $\mathrm{m}$

eye[i] $=$ rand $O \%(m+1)$;

// cycljl is in the range of 0 to $m$

cout $<<$ exe[j] $<-\infty<$ exye[j] <endl;

for $(\mathrm{i}=0, \mathrm{j}=0 ; \mathrm{i}<\mathrm{ns}, \mathrm{j}<\operatorname{\text {ac}} ; \mathrm{i}++, \mathrm{j}++)$

if(exe[i] = sxe[i] \&\& eyeli] = =sye[i])

/hif coordinate position of camera sensor and scalar sensor matches

$\operatorname{exe}[j]=\operatorname{exe}[j]+1$

fincrement $x$-coordinate position of camera sensor by 1 to avoid overlapping of

coordinate positions

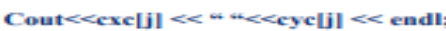

Cout $<<$ sxe[i] $\ll$ " $"<<$ syc|il $\ll$ endl;

3

Fig. 6. Data Generation Procedure 


\subsection{Results and Elucidation}

\section{(i) Repercussion of $\mathrm{ns}$}

During experimentation, the $n s$ has been altered and its impact is studied on number of cameras actuated (nca) as illustrated in Table 2 while keeping number of cameras deployed at 300. This is observed that with accession in $n s$, the nca gets escalated for all the approaches because more count of scalars come under the hold of occurring event. Moreover, since the number count of event detecting scalars rises, the number of event ensnaring SLs also hike resulting in gradual increase in $n c a$ values. Further, the results in Table 2 clearly show that the $n c a$ value is the minimum in our $H S$-ASLE approach. The minimal $n c a$ value is attained at 52 .

Table 2. ns versus nca

\begin{tabular}{|c|c|c|c|}
\hline ns & $\begin{array}{c}\text { nca } \text { in } \\
\text { DCA-SC }\end{array}$ & $\begin{array}{c}\text { nca in } \\
\text { DCCA-SM }\end{array}$ & $\begin{array}{c}\text { nca in } \\
\text { HS-ASLE }\end{array}$ \\
\hline 250 & 82 & 79 & $\mathbf{5 2}$ \\
\hline 300 & 93 & 91 & 56 \\
\hline 350 & 97 & 94 & 57 \\
\hline 400 & 103 & 100 & 58 \\
\hline 450 & 106 & 104 & 63 \\
\hline 500 & 110 & 106 & 67 \\
\hline 550 & 112 & 107 & 70 \\
\hline 600 & 116 & 111 & 72 \\
\hline 650 & 119 & 117 & 76 \\
\hline 700 & 125 & 119 & 81 \\
\hline
\end{tabular}

Table 3. ns versus cr

\begin{tabular}{|c|c|c|c|}
\hline ns & $\begin{array}{c}\text { cr in } \\
\text { DCA-SC }\end{array}$ & $\begin{array}{c}\text { cr in } \\
\text { DCCA-SM }\end{array}$ & $\begin{array}{c}\text { cr in } \\
\text { HS-ASLE }\end{array}$ \\
\hline 250 & 0.61 & 0.62 & 0.80 \\
\hline 300 & 0.63 & 0.65 & 0.81 \\
\hline 350 & 0.64 & 0.66 & 0.83 \\
\hline 400 & 0.66 & 0.68 & 0.86 \\
\hline 450 & 0.68 & 0.69 & 0.88 \\
\hline 500 & 0.69 & 0.71 & 0.89 \\
\hline 550 & 0.70 & 0.73 & 0.91 \\
\hline 600 & 0.72 & 0.74 & 0.92 \\
\hline 650 & 0.73 & 0.76 & 0.94 \\
\hline 700 & 0.75 & 0.77 & $\mathbf{0 . 9 5}$ \\
\hline
\end{tabular}


Table 4. ns versus rr

\begin{tabular}{|c|c|c|c|}
\hline ns & $\begin{array}{c}\text { rr in } \\
\text { DCA-SC }\end{array}$ & $\begin{array}{c}\text { rr in } \\
\text { DCCA-SM }\end{array}$ & $\begin{array}{c}\text { rr in } \\
\text { HS-ASLE }\end{array}$ \\
\hline 250 & 0.60 & 0.61 & $\mathbf{0 . 3 0}$ \\
\hline 300 & 0.62 & 0.64 & 0.32 \\
\hline 350 & 0.64 & 0.65 & 0.33 \\
\hline 400 & 0.66 & 0.66 & 0.35 \\
\hline 450 & 0.67 & 0.67 & 0.37 \\
\hline 500 & 0.68 & 0.68 & 0.38 \\
\hline 550 & 0.70 & 0.71 & 0.39 \\
\hline 600 & 0.73 & 0.73 & 0.41 \\
\hline 650 & 0.74 & 0.75 & 0.42 \\
\hline 700 & 0.76 & 0.77 & 0.44 \\
\hline
\end{tabular}

Table 5. ns versus eeca

\begin{tabular}{|c|c|c|c|}
\hline ns & $\begin{array}{c}\text { eeca in } \\
\text { DCA-SC } \\
\text { (in joule) }\end{array}$ & $\begin{array}{c}\text { eeca in } \\
\text { DCCA-SM } \\
\text { (in joule) }\end{array}$ & $\begin{array}{c}\text { eeca in } \\
\text { HS-ASLE } \\
\text { (in joule) }\end{array}$ \\
\hline 250 & 104.96 & 101.12 & $\mathbf{6 7 . 8 4}$ \\
\hline 300 & 119.04 & 116.48 & 71.68 \\
\hline 350 & 152.37 & 120.32 & 72.96 \\
\hline 400 & 131.84 & 128.00 & 74.24 \\
\hline 450 & 135.68 & 133.12 & 80.64 \\
\hline 500 & 140.80 & 135.68 & 85.76 \\
\hline 550 & 143.36 & 136.96 & 89.60 \\
\hline 600 & 148.48 & 142.08 & 92.16 \\
\hline 650 & 152.32 & 149.76 & 97.28 \\
\hline 700 & 160.00 & 152.32 & 103.68 \\
\hline
\end{tabular}

Similarly, Table 3 depicts that with escalation in $n s$, the coverage ratio escalates for all methods, and is realized to be highest for HS-ASLE owing to efficient determination of SLs. Since in alternate virtual sub-compartments one and two SL(s) get(s) selected, so each alternative sub-compartmental $S L$ enhances the possibility of greater number of event detecting SLs so that more cameras get informed and this leads to greater camera actuation, thereby enhancing the $c r$ value. The maximal cr value is attained at 0.95 .

Table 4 layouts the variation of redundancy ratio $(r r)$ against $n s$ values. It is observed from the table that the $r r$ hikes with rise in nsd since with hike in count of scalars, higher count of scalars come under the purview of occurring event. However, $r r$ values are attained to be the least in the proffered $H S-A S L E$ approach, thereby, ensuring minimal repeated data transmittal in $H S-A S L E$. The minimal $r r$ value is attained at 0.30 in $H S-A S L E$. Besides, the activation of least number of cameras in the proffered $H S$-ASLE scheme leads to 
least value for eeca as comparison to the other methods [1,2] as illustrated in Table 5. The least eeca value is attained as 67.84 joule in $H S$-ASLE.

\section{(ii) Repercussion of $n c$}

We have changed the $n c$ and conjointly studied its repercussion on $n c a$ as illustrated in Table 6 while keeping number of scalars fixed at 400. With accretion in $n c, n c a$ gets escalated for all the strategies because more number of camera sensors come within the range of ongoing event. Further, the results in Table 6 clearly shows that the $n c a$ value is the minimum in our HS-ASLE approach at 59.

Similarly, Table 7 depicts that with hike in $n c$, the coverage ratio (cr) escalates for all the methods as higher count of cameras arrive under the ambit of occurring event, and $\mathrm{cr}$ is attained to be the maximal for $H S-A S L E$ owing to efficient determination of $S L s$ and more cameras get informed due to increase in $n c$ and this leads to greater camera actuation, thereby enhancing the $c r$ value. The maximum value of $c r$ is attained as 0.96 in case of $H S$ ASLE.

Table 8 portrays the variation of redundancy ratio $(r r)$ against $n c$ values. It is observed from the table that the $r r$ hikes with rise in $n c$ as with hike in count of cameras, higher count of cameras come under the purview of occurring event. However, $r r$ values are attained to be the least at 0.31 in the proffered $H S$-ASLE approach, thereby, ensuring minimal repeated data transmittal in $H S$-ASLE. Besides, the count of camera activation in the proffered scheme gives rise to least value for eeca (i. e. 75.52 joule) in comparison to the other two methods $[1,2]$ as illustrated in Table 9.

Table 6. nc versus nca

\begin{tabular}{|c|c|c|c|}
\hline nc & $\begin{array}{c}\text { nca in } \\
\text { DCA-SC }\end{array}$ & $\begin{array}{c}\text { nca in } \\
\text { DCCA-SM }\end{array}$ & $\begin{array}{c}\text { nca in } \\
\text { HS-ASLE }\end{array}$ \\
\hline 150 & 91 & 95 & $\mathbf{5 9}$ \\
\hline 170 & 102 & 105 & 62 \\
\hline 190 & 108 & 111 & 66 \\
\hline 210 & 116 & 119 & 69 \\
\hline 230 & 114 & 117 & 75 \\
\hline 250 & 118 & 119 & 77 \\
\hline 270 & 121 & 125 & 81 \\
\hline 290 & 125 & 129 & 87 \\
\hline 310 & 127 & 132 & 90 \\
\hline 330 & 130 & 134 & 87 \\
\hline
\end{tabular}

Table 7. nc versus cr

\begin{tabular}{|c|c|c|c|}
\hline nc & $\begin{array}{c}\text { cr in } \\
\text { DCA-SC }\end{array}$ & $\begin{array}{c}\text { cr } \text { in } \\
\text { DCCA-SM }\end{array}$ & $\begin{array}{c}\text { cr } \text { in } \\
\text { HS-ASLE }\end{array}$ \\
\hline 250 & 0.63 & 0.65 & 0.78 \\
\hline 300 & 0.64 & 0.66 & 0.79 \\
\hline 350 & 0.68 & 0.69 & 0.80 \\
\hline 400 & 0.70 & 0.73 & 0.82 \\
\hline 450 & 0.73 & 0.77 & 0.85 \\
\hline
\end{tabular}




\begin{tabular}{|l|l|l|l|}
\hline 500 & 0.76 & 0.79 & 0.87 \\
\hline 550 & 0.79 & 0.80 & 0.89 \\
\hline 600 & 0.81 & 0.84 & 0.93 \\
\hline 650 & 0.82 & 0.85 & 0.94 \\
\hline 700 & 0.84 & 0.88 & $\mathbf{0 . 9 6}$ \\
\hline
\end{tabular}

Table 8. nc versus rr

\begin{tabular}{|c|c|c|c|}
\hline nc & $\begin{array}{c}\text { rr in } \\
\text { DCA-SC }\end{array}$ & $\begin{array}{c}\text { rr in } \\
\text { DCCA-SM }\end{array}$ & $\begin{array}{c}\text { rr in } \\
\text { HS-ASLE }\end{array}$ \\
\hline 250 & 0.59 & 0.60 & $\mathbf{0 . 3 1}$ \\
\hline 300 & 0.60 & 0.62 & 0.34 \\
\hline 350 & 0.62 & 0.63 & 0.36 \\
\hline 400 & 0.64 & 0.65 & 0.38 \\
\hline 450 & 0.66 & 0.67 & 0.40 \\
\hline 500 & 0.67 & 0.68 & 0.43 \\
\hline 550 & 0.68 & 0.70 & 0.44 \\
\hline 600 & 0.70 & 0.72 & 0.45 \\
\hline 650 & 0.71 & 0.73 & 0.47 \\
\hline 700 & 0.72 & 0.75 & 0.49 \\
\hline
\end{tabular}

Table 9. nc versus eeca

\begin{tabular}{|c|c|c|c|}
\hline nc & $\begin{array}{c}\text { eeca in } \\
\text { DCA-SC } \\
\text { (in joule) }\end{array}$ & $\begin{array}{c}\text { eeca in } \\
\text { DCCA-SM } \\
\text { (in joule) }\end{array}$ & $\begin{array}{c}\text { eeca in } \\
\text { HS-ASLE } \\
\text { (in joule) }\end{array}$ \\
\hline 150 & 116.48 & 121.60 & $\mathbf{7 5 . 5 2}$ \\
\hline 170 & 130.56 & 131.25 & 79.36 \\
\hline 190 & 138.24 & 142.08 & 84.48 \\
\hline 210 & 148.48 & 148.75 & 88.32 \\
\hline 230 & 145.92 & 149.76 & 96.00 \\
\hline 250 & 151.04 & 152.32 & 98.56 \\
\hline 270 & 154.88 & 156.25 & 103.68 \\
\hline 290 & 160.00 & 165.12 & 107.52 \\
\hline 310 & 162.56 & 168.96 & 111.36 \\
\hline 330 & 166.40 & 171.52 & 115.20 \\
\hline
\end{tabular}

\section{Conclusions and Future Scope}

This paper reports a novel scheme namely, HS-ASLE that involves the intellectual election of scalar leaders that transfer information pertaining to the events to the concerned camera sensors and the appropriate cameras under go activation so as to cover the geographic event zone efficiently in a multi-event occurrence scenario. Experiments have been conducted to validate the execution of the suggested HS-ASLE approach in comparison with two other recent methods. The numerical results attained from the investigation establishes the superiority of the proffered method in terms of nca, cr, rr and eeca. 
While varying the ns, the nca and rr values are attained at 52 and 0.30 respectively in our proffered $H S$-ASLE approach. In this context, $c r$ is the highest in proposed scheme at 0.95 and the eeca is the minimal at 67.84 joule. Similarly, we have changed the $n c$ and speculated its repercussion on the other two methods. It is seen that the minimal $n c a$ and rr values are obtained in our proposed $H S$-ASLE approach at 59 and 0.31 respectively. Similarly, the cr is attained at 0.96 in $H S$-ASLE. Further, the eeca value is the lowest at 75.52 joule in proposed HS-ASLE. Our proposed model can be mapped into three dimensional space as a future direction of our investigation.

\section{REFERENCES}

1. A. Newell and K. Akkaya, "Distributed Collaborative Camera Actuation for Redundant Data Elimination in Wireless Multimedia Sensor Networks", Ad Hoc Networks, Elsevier, vol. 9, no. 4, pp. 514-527, 2011.

2. W. Luo, Q. Lu, J. Xiao, "Distributed Collaborative Camera Actuation Scheme based on Sensing-region Management for Wireless Multimedia Sensor Networks”, Int. J. Distrib. Sensor Networks, vol. 2012, pp. 1-14, Article ID: 486163, 2012.

3. S. B. B. Priyadarshini and S. Panigrahi, "A Non-heuristic Approach for Minimizing the Energy and Power Consumption in Wireless Multimedia Sensor Networks", Proceedings of International Conference on Computational Intelligence and Networks (CINE), Published in IEEE Xplore Digital Library, Bhubaneswar, 12-13 Jan. 2015, pp. 104-109.

4. S. B. B. Priyadarshini and S. Panigrahi, "Centralised cum sub-centralised scheme for multi-event coverage and optimum camera activation in wireless multimedia sensor networks", Special Issue on Creating a Smarter Environment through Advancement of Communication Systems, Networks and Applications, IET Networks, Published in IET and IEEE Xplore Digital Library, vol. 4, no. 6, pp. 314-328, 2015.

5. D. Zorbas, D. Glynos, P. Kotzanikolaou, P., "Christos Douligeris, Solving Coverage Problems in Wireless Sensor Networks using Cover sets", Ad Hoc Networks, Elsevier Science publishers, vol. 8, no. 4, pp. 400-415, 2010.

6. J. Wang, C. Niu, R. Shen, "Priority-based Target Coverage in Directional Sensor Networks using a Genetic Algorithm", Computers and Mathematics with Applications, Elsevier, vol. 57no. 11-12, pp. 1915-1922, 2009.

7. S. S. Ram, D. Manjunath, S. K. Iyer, S. K., D. Yogeshwaran, On the Path Coverage Properties of Random Sensor Networks. IEEE TRANSACTIONS ON MOBILE COMPUTING, vol. 6, no. 5, pp. 494-506, 2007.

8. A. Girault, "Elimination of Redundant Messages with a Two-pass Static Analysis Algorithm", Parallel Computin, Elsevier, vol. 28, no. 3, pp. 433-453, 2002.

9. A. Ghaddar ,T. Razafindralambo, S. Tawbi and A. Hijazi (2010 june). "Algorithm for Data Similarity Measurements to Reduce Data Redundancy in Wireless Sensor Networks", Proceedings of IEEE International Symposium on A World of Wireless, Mobile and Multimedia Networks (Wow Mom), ISBN: 978-1-4244-7264-2, Montreal, QC, Canada., June 14-june 17, 2010., 
10. S. Toumpis and T. Tassiulas, T., "Optimal Deployment of Large Wireless Sensor Networks", IEEE Transactions on Information Theory”, vol. 52, no. 7, pp. 2935-2953, 2006.

11. X. Han, X. Cao, E. Lloyd and C. Shen, "Deploying Directional Sensor Networks with Guaranteed Connectivity and Coverage", Proceeding of $5^{\text {th }}$ Annual IEEE Communications Society Conference on Sensor, Mesh and Adhoc Communications and Networks, San Francisco, CA, June 16 - june 20, 2008, pp. 153-159

12. A. Abdullah, A. Shalabi, M. Manaf, "DKCS- An Efficient Dynamic K- coverage Scheduling Algorithm for Wireless Sensor Networks" Proceedings of Telecommunication Technologies (ISST) 2012 International Symposium, IEEE, Kuala Lumpur, 26 ${ }^{\text {th }}-28^{\text {th }}$ November, 2012, pp. 94-99.

13. Y. Xieliu, S. Fang, "Effect of Field of view on the Accuracy of Camera Calibration", Optik -International Journal for Light and Electron Optics, Elsevier, vol. 125, no. 2, pp. 844-849, 2014.

14. Q. Zhao, Luo, M. Gurusamy, "Lifetime maximization for connected target coverage in wireless sensor networks”, IEEE/ACM Trans. Netw., vol. 16, no. 6, pp. 1378-1391, 2008. 